\title{
An Efficient Fingerprint Matching System for Low Quality Images
}

\author{
Zin Mar Win \\ Department of Software Technology \\ University of Computer Studies \\ Yangon, Myanmar
}

\author{
Myint Myint Sein \\ Department of Research and Development \\ University of Computer Studies \\ Yangon, Myanmar
}

\begin{abstract}
Fingerprint-based identification is one of the most well-known and publicized biometrics for personal identification. It remains a reliable, efficient and commonly accepted biometric. In this paper, a fingerprint recognition system for identifying the low quality fingerprint images on Myanmar National Registration Cards (NRCs) is developed. Traditional minutia based approach is not robust to poor quality fingerprint images. In proposed system, ridge feature-based approach for fingerprint recognition using contextual filter and single pass thinning algorithm is developed. The input image is preprocessed and gabor filtering is applied for ridge line enhancement. The system extracts the ridge line features from the skeleton image derived from single pass thinning algorithm and it is compared to the database using Euclidean distance metric. The effectiveness of the proposed system can be confirmed through the experimental results.
\end{abstract}

\section{General Terms}

Pattern Recognition, Image Processing, Biometric

\section{Keywords}

Fingerprint, Fingerprint Recognition, Gabor filter, Single Pass thinning, Histogram matching

\section{INTRODUCTION}

Biometrics is formed from the person's selected unique physical attributes which may be applied for the purpose of automated personal identification [1]. Biometrics is a rapidly evolving technology that has been widely used in forensics, such as criminal identification and prison security, and has the potential to be widely adopted in a very broad range of civilian applications such as Banking security, Physical access control, Information system security, customs and immigration. Fingerprints have been routinely used as a method for person identification for more than a century [2].

Fingerprints of any individual are unique (even in the case of identical twins), remain the same over lifetime, and are easy to collect. A pattern of ridges, valleys and minutiae can be extracted from the fingerprint image. A fingerprint pattern is composed of a sequence of ridges and valleys which generally run parallel to each other in fingerprint. The ridges are dark lines while the valleys are the light areas between the ridges. The underlying ridge structure pattern can be analyzed on a global and local level. These are the features of fingerprint. The global features mainly give an overall characteristic of the finger. A global feature normally provides a special pattern of ridges and valleys including singularities or singular point (SP). The most used singularities are core and delta. While the core is usually defined as a point on the inner most ridge, the delta is known as the center point where three different flows meet. The SP provides important information used for fingerprint classification [3,4] as shown in Figure 1, fingerprint matching [5,6] and fingerprint alignment [7,8]. Generally, fingerprints can be classified into five classes: left loop, right loop, whorl, arch and tented arch. Major concept used in local representations of fingerprints is finger ridges. Minutiae are minute details of the fingerprint [8] and they are shown in Figure 2. Minutiae are the locations where a ridge becomes discontinuous.

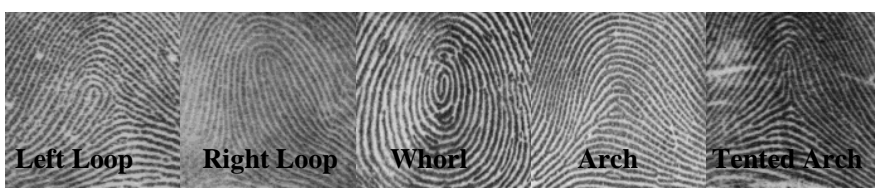

Figure 1. Fingerprint Classes

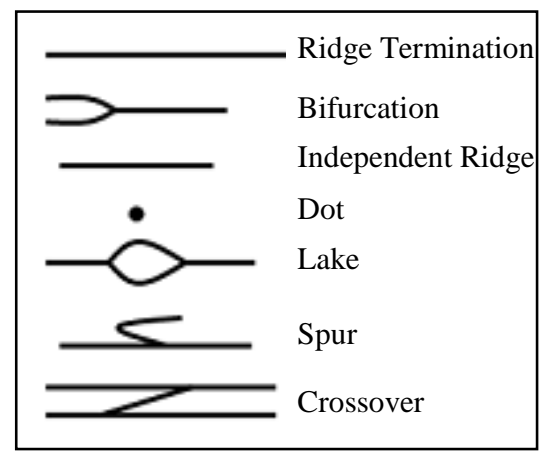

Figure 2. Minutiae Types

In forensics, fingerprint images can be classified in two categories: fingerprint impressions and latent fingermarks. Fingerprint impressions are obtained either by scanning the inked impressions on paper or by using scanning devices [9]. Latent fingerprints are obtained from crime scenes and usually come from small portion of friction ridge skins. The qualities of fingerprint images are mainly depend on the acquisition devices.

The different kinds of fingerprint images are described in Figure 3. The high quality images acquired by live-scan device, fingerprint inked impression on paper and low quality image with fabric background on NRC card are shown. 


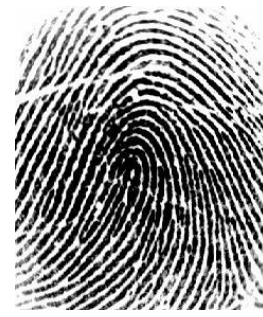

(a)

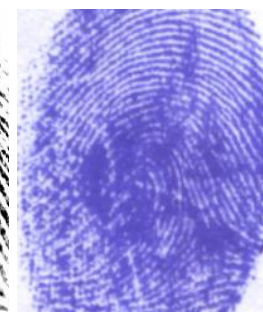

(b)

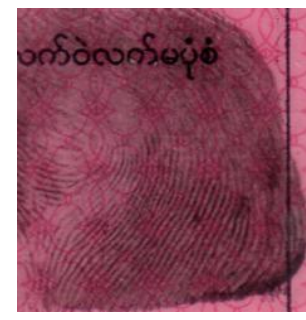

(c)
Figure 3. Different kinds of fingerprint images(a) live-scan (b) inked fingerprint on paper (c) latent print on NRC card

A fingerprint recognition system can be used for both verification and identification. In verification, the system compares an input fingerprint to the "enrolled" fingerprint of a specific user to determine if they are from the same finger (1:1 match). In identification, the system compares an input fingerprint with the prints of all enrolled users in the database to determine if the person is already known under a duplicate or false identity $(1: \mathrm{N}$ match).

Fingerprint matching means matching of two fingerprint images with respect to certain features like minutiae, ridges, etc of two images. The feature based matching is an appropriate method of matching and provides better results for majority of fingerprints, but there are a number of fingerprints of low quality which could not be identified easily by these methods [10]

Several methods of automatic fingerprint identification have been proposed in the literature. Minutiae based approach often gives satisfactory results for good quality images. But if, the quality of the image is poor, then minutiae extraction is a very difficult task and often gives incorrect results that are not acceptable for real time authentication applications. The minutiae sets may suffer from false, missed, and displaced minutiae, caused by poor fingerprint image quality and imperfections in the minutiae extraction stage [11].

Another class of finger-print matching algorithms doesn't use the minutiae features of the fingerprint. These methods usually match features extracted from the image by means of certain filtering or transform operations; hence they are named ridge feature-based methods. The 2D wavelet decomposition on $\mathrm{J}$ octaves of the image is used as the features for recognition [12]. These approaches require less preprocessing or post processing effort than minutiae-based methods. While minutiae-based methods normally require a minutiae location process [13] [14], imagebased methods match two fingerprint images directly, based on their texture features. The general overview of the fingerprint identification system is as shown in Figure 4.

In this paper, we propose an ridge feature-based approach towards fingerprint recognition. The fingerprint images are matched based on ridge line features extracted by using contextual filtering and two pass thinning. Histogram approach is applied for finger matching to ensure the system accuracy. The proposed system can recognize not only the fingerprint acquired from device but also the low quality fingerprint image from inked-printed images on paper.

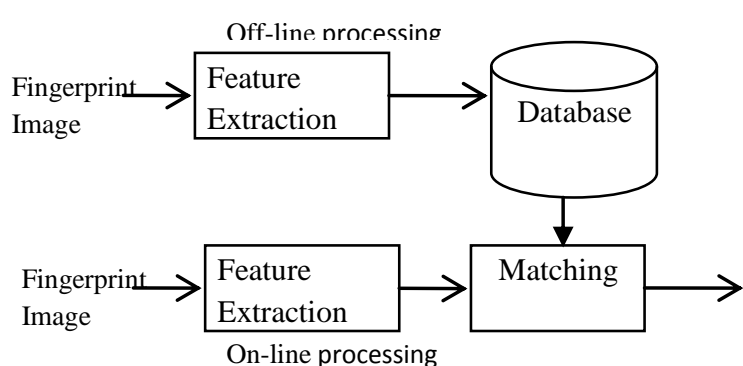

Figure 4.Overview of Fingerprint Identification

The rest of the paper is organized as follows: section 2 reports related works. In section 3 and 4 , the overview of the proposed system and the preprocessing of fingerprint image are described. Section 5 and 6 present feature extraction and matching. Section 7 is experimental results. Finally, in section 8 , the concluding remarks are given.

\section{RELATED WORKS}

There has been a lot of work in various types of fingerprint identification. A couple of works carried out in this direction can be found. The existing systems perform classification and identification of fingerprint. The fingerprints can be classified into five different types according to the global ridge features in order to reduce the search time and computational complexity. Masayoshi Kamijo's Classification approach [15]: It is an ANN based approach, where a neural network for the classification of fingerprint images is constructed, which can classify the complicated fingerprint images. It uses a two-step learning method to train the four layered neural network which has one sub-network for each category. It carries out the principal component analysis (PCA) with respect to the unit values of the second hidden layer and also studies the fingerprint classification state represented by the internal state of the network. Consequently, the method confirms that the fingerprint patterns are roughly classified into each category in the second hidden layer and the effectiveness of the two-step learning process. However, in case of larger data sets this method can be found to give limited results.

Karu and Jain's Classification approach [16]: In this approach, it initially finds the ridge direction at each pixel of an input fingerprint image. Then the algorithm extracts global features such that singular points (cores and deltas) in the fingerprint image and performs the classification based on the number and locations of the detected singular points. Here, the singular point(s) detection is an iterative regularization process until the valid singular points are detected. If the images are of poor quality, the algorithm classifies those images as unknown types based on some threshold values. However, the algorithm can detect the labeled images with high quality.

With the development of fingerprint identification, the state of the art application use the fingerprint ridge line features such as minutiae point and texture feature in order to obtain the improved fingerprint recognition system. Dadgostar et al., [17] initiated a fingerprint identification method in which features are extracted using Gabor filter and Recursive Fisher Linear Discriminant (RFLD) algorithms. Features extracted by Gabor filter are usually high dimensional. To reduce the dimension and also to extract more discriminant features, RFLD algorithm is used. Umair 
Mateen Khan et al., [18] discussed a fingerprint matching criteria. The features extracted from both minutiae based method and wavelet transformation based method are combined to get better results.

Zhou Weina et al., [19] described an algorithm combining wavelet transform with prewitt edge detection for fingerprint verification. The fingerprint verification system is based on wavelet's supply of detail information and prewitt edge detection's stable characteristics in translation, scaling and rotation. Leon et al., [20] developed two algorithms for image enhancement and also, the invariant moments in verification phase. Fingerprint verification is considered using a combination of Fast Fourier Transform (FFT) and Gabor filters by image enhancement. A thinning algorithm is applied to get an image with the minimum thickness of one pixel. The feature vector is generated with the distance between minutiae, angle between minutiae and coordinates. Ujjal Kumar Bhowmik et al., [21] derived a minutiae matching algorithm using the smallest minimum sum of closest Euclidean Distance. The algorithm extracts matched minutiae pairs from input and template fingerprints, corresponding rotation angle and empirically chosen statistical threshold values. The method reduces the effect of non-linear distortion using only the minutiae location. Mohammad Sadegh Helfroush and Mohsen Mohammadpour, [22] developed a method for fingerprint verification system. The method is based on the fusion of spectral and directional features which improves the performance in terms of EER and is simple so that the verification speed is high. The method does not need any reference point and preprocessing. Features are extracted using all pixels of fingerprint image.

Alessandra Paulino et al., [23] developed the latent fingerprint matching with fusion of manually marked and automatically extracted minutiae. The system matches latent to full fingerprint matching accuracy by combining manually marked (ground truth) minutiae with extracted minutiae. The fingerprint orientation fields are reconstructed from minutiae and singular points. The resulted orientation image is enhanced using Gabor filter approach. The extracted minutiae are compared against the minutiae in Rolled fingerprint.

\section{SYSTEM OVERVIEW}

In proposed system, there are enrollment phase and authentication phase for fingerprint recognition. In enrollment phase, the fingerprint image acquired from NRC card is preprocessed and features are extracted. The fingerprint on NRC card is very low quality with complex background and difficult to extract features properly. Therefore, in order to be the narrow area with good ridge features around the center area, the center point or core point of the fingerprint is manually selected and cropping is done around the center point. To improve the clarity of the ridge lines, the enhancement of fingerprint is performed. The features are ridge skeleton image and stored into the database. For authentication phase, the input fingerprint is preprocessed, enhanced and the ridge skeleton image is extracted from the enhanced image. The feature points are extracted from the skeleton image and matched with the fingerprint image from the database using the Euclidean distance metric. The input image is recognized to belong to one of the fingerprints of the database, if the smallest distance between the input image and stored image is lesser than a threshold value. If the smallest distance is not lesser than the fixed threshold then the input image doesn't belong to any of the fingerprints.

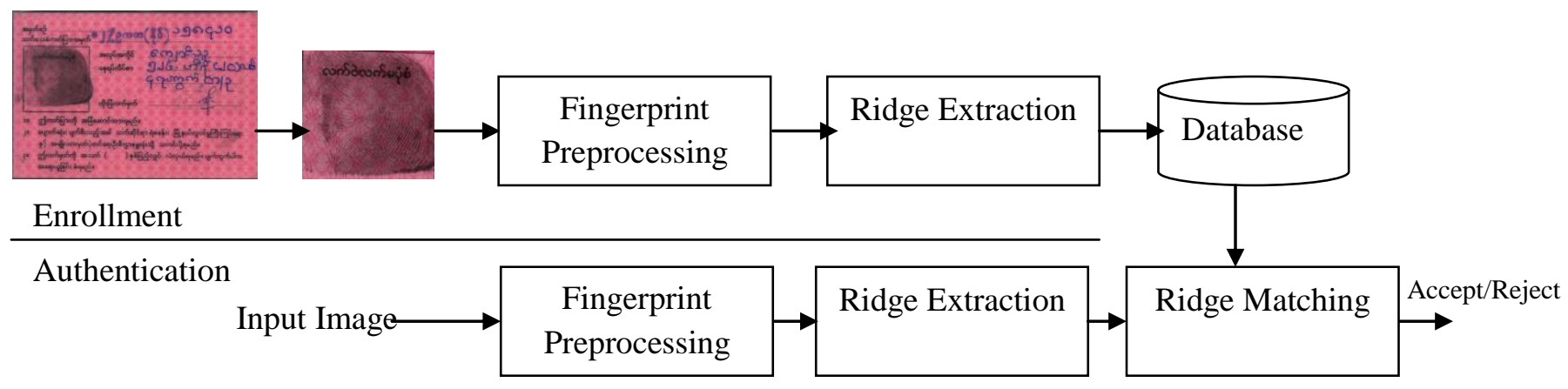

Figure 5. System Flow Chart

\section{FINGERPRINT PREPROCESSING}

The performance of a fingerprint image-matching algorithm depends heavily on the quality of the input fingerprint images. It is very important to acquire good quality images but in practice a significant percentage of acquired images is of poor quality due to some environmental factors or user's body condition. The main steps involved in the image preprocessing include: noise removal, region cropping, ridge orientation detection and enhancement.

\subsection{Noise removal}

The fingerprint images acquired from NRC card have noise and complex fabric background. The fingerprint image is segmented to extract the region of interest (ROI) which contains the desired fingerprint impression. The original fingerprint and the extracted image from background after removing noise are shown in Figure 6. 


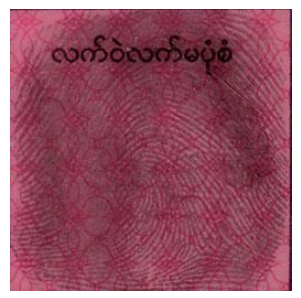

(a)

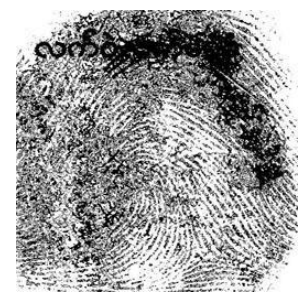

(b)
Figure 6.(a) fingerprint from NRC card (b) Extracted image from background

\subsection{Region Cropping}

To reduce the template size, only an area or region with unique pattern of curvature is needed to store the database. However, the common center point for both the registered and input images is needed. In proposed system, the fingerprint center point is manually selected for region cropping. Keeping the center point, the image of size $\mathrm{w} \mathrm{x} \mathrm{w}$ is cropped and further processing is done with the cropped portion of the image. The cropped image and histogram equalized image are shown in Figure 7.

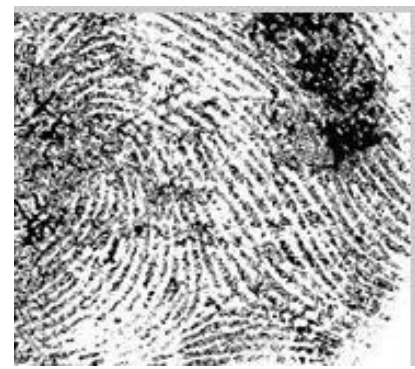

(a)

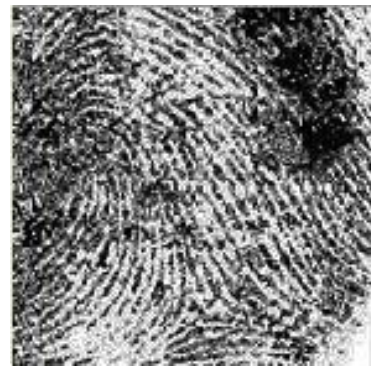

(b)
Figure 7(a) Cropped Image (b) After histogram equalization

\subsection{Ridge Orientation Detection}

The term orientation image often refers to the determination of local ridge orientation in the fingerprint image. There have been proposed some methods for ridge orientation computation. Matched-filter approach [24], methods based on the highfrequency power in 3-D spaces [25] and method based on 2-D spectral estimation methods [26]. Due to the limited number of fixed numbers of orientations, these methods do not generate much accuracy. Gradient-based method was introduced in [27] and has been utilized by some researchers. In proposed system, gradient-based approach is used to detect fingerprint orientation.

The orientation of a pixel in a image can be expressed as a vector $\left[G_{x}(x, y), G_{y}(x, y)\right]^{T}$, which is defined as [27]:

$$
\left[\begin{array}{l}
G_{x}(x, y) \\
G_{y}(x, y)
\end{array}\right]=\nabla I(x, y)=\left[\begin{array}{l}
\frac{\partial(I(x, y))}{\partial x} \\
\frac{\partial(I(x, y))}{\partial y}
\end{array}\right]
$$

where $I(x, y)$ is the gray-scale fingerprint image. Commonly, a main orientation in a block can be decided by the gradient vector of most pixels. So the gradient vector of all pixels in a local area can be used to estimate the local ridge orientation. However, gradients cannot directly be accumulated in local area since opposite gradient vectors will cancel each other, although they denote the same ridge orientation. Method in [27, 28] uses a method to double the angles of the gradient vectors before averaging, in this way opposite gradient vectors will point to the same direction. In [29], not only the angle is doubled, but also the length of the gradient vectors is squared.

In this paper, a convenient way to estimate the local ridge orientation is proposed. We first adjust equation (2) as follows:

$$
\left[\begin{array}{l}
G_{x}(x, y) \\
G_{y}(x, y)
\end{array}\right]=\operatorname{sign}\left(\frac{\partial(I(x, y))}{\partial x}\right)\left[\begin{array}{l}
\frac{\partial(I(x, y))}{\partial x} \\
\frac{\partial(I(x, y))}{\partial y}
\end{array}\right]
$$

It is confirmed that the first element in gradient vector is positive in equation (2). The opposite gradient vector will reinforce each other while averaging. The local ridge orientation can be obtained in the following steps:

1. Accumulate the gradient of $x$ and $y$ axis using equations (3) and (4):

$$
\begin{aligned}
G_{x} & =\sum_{W} G_{x}(x, y) \\
G_{Y} & =\sum_{W} G_{y}(x, y)
\end{aligned}
$$

2. Mutative orientation between ridges and valleys, which is perpendicular to ridge orientation, can be computed as equation (5):

$$
\psi(i, j)=\arctan \left(G_{Y} / G_{X}\right)
$$

3. Using equation (6), local ridge orientation is obtained.

$$
\theta(i, j)= \begin{cases}\psi(i, j)+0.5 \pi & \psi(i, j) \leq 0 \\ \psi(i, j)-0.5 \pi & \psi(i, j)>0\end{cases}
$$

where $(i, j)$ is the block number in fingerprint image, $\theta(i, j)(\theta \in$ $(-0.5 \pi, 0.5 \pi))$ is the corresponding ridge orientation, and $W$ denotes the range of block.

Ridge frequency is another intrinsic property of the fingerprint. The most commonly used method for computing ridge frequency is based on the projection sum taken [28]. Oriented fingerprint image is shown in Figure 8(a).

\subsection{Enhancement}

Fingerprint image enhancement is to make the image clearer for easy further operations. Since the fingerprint images acquired from the NRC cards are not assured with perfect quality, enhancement methods, for increasing the contrast between ridges and furrows and for connecting the false broken points of ridges due to insufficient amount of ink, are very useful to keep a higher accuracy to fingerprint recognition. 
For fingerprint image on NRC card, it is very difficult to obtain a reliable orientation field based on the image itself. Also, the ridge frequency estimated from the latent image is not reliable. Therefore, we applied the enhancement process proposed in [28] using the orientation image obtained in earlier step.

The gabor filtering is applied for fingerprint enhancement in [28]. The ridges and valleys in a small local neighborhood have well defined local frequency and local orientation properties. A set of band pass filters can remove the undesired noise and preserve true ridge structures. Gabor filters have both frequency-selective and orientation-selective properties and have optimal joint resolution in both spatial and frequency domains. Gabor filters are used to remove the noise and preserve true ridge/valley structures. The filter applied at each pixel $[x, y]$ has the form:

$$
g(x, y: \theta, f)=e^{\left(\frac{-(x+y)^{2}}{2 \delta^{2}}\right)} \cos [2 \pi \cdot f \cdot(x \cdot \sin \theta+y \cdot \cos \theta)](7)
$$

where $\theta$ and $f$ are the corresponding local orientation and frequency. Enhanced image is shown in Figure 8(b).

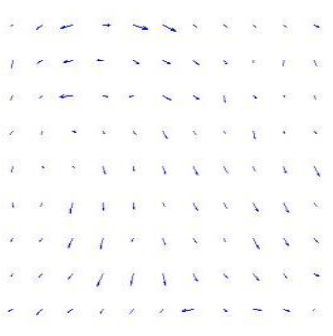

(a)

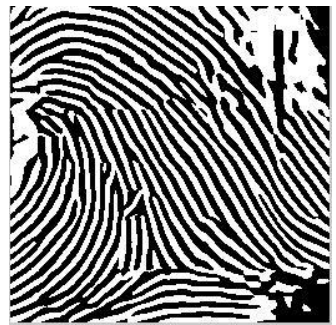

(b)
Figure 8(a) Orientation Image (b) Enhancement Image

\section{FEATURE EXTRACTION}

Feature extraction is concerned with the quantification of ridge line characteristics. The proposed system utilizes the features of the skeleton image of the fingerprint. The skeleton image is onepixel-wide ridge, which is traced in the thinned image. Single pass thinning algorithm is applied for obtaining skeleton fingerprint.

\subsection{Single Pass Thinning}

Single pass thinning algorithm uses both flag map amd smoothing templates for boundary pixel deletion [30]. The skeleton produced by this algorithm is not only one-pixel thick, perfectly connected, well-defined, but also has the desired property of handling boundary noise. In thinning algorithm, the image and the flag map are used together to decide on which pixel to delete. When examining a pixel and its neighbours to decide if it is to be flagged as a boundary pixel, both the flag and the results from the last iteration are used in the decision-making process.

The symbols $\mathrm{P}_{0}, \mathrm{P}_{1}, \mathrm{P}_{2}, \mathrm{P}_{3}, \mathrm{P}_{4}, \mathrm{P}_{5}, \mathrm{P}_{6}, \mathrm{P}_{7}$ and $\mathrm{P}_{8}$, shown in Figure 9 (a), represent the pixel $P[i][j]$ in the bitmap and its neighbours. The flag map is used for flagging those pixels that will eventually be deleted. The size of the flag map is the same as that of the image. For an $n \times m$ image, the size of the flag map will also be $\mathrm{n} \times \mathrm{m}$. The symbols $\mathrm{Q}_{0}, \mathrm{Q}_{1}, \mathrm{Q}_{2}, \mathrm{Q}_{3}, \mathrm{Q}_{4}, \mathrm{Q}_{5}, \mathrm{Q}_{6}, \mathrm{Q}_{7}$ and $\mathrm{Q}_{8}$, as shown in Figure 9(b), correspond to $\mathrm{P}_{0}, \mathrm{P}_{1}, \mathrm{P}_{2}, \mathrm{P}_{3}, \mathrm{P}_{4}, \mathrm{P}_{5}, \mathrm{P}_{6}, \mathrm{P}_{7}$ and $\mathrm{P}_{8}$ respectively. Initially, all pixels in the flag map are set to 1 . The value will be changed to 0 as soon as that pixel is flagged.

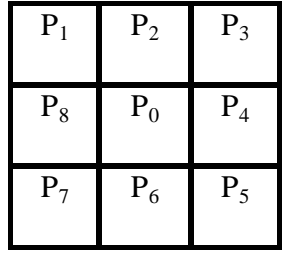

(a)

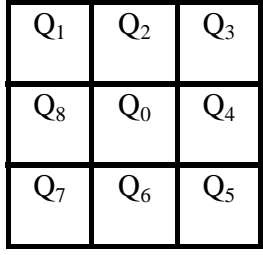

(b)
Figure 9(a)Local pixel notation of bitmap (b) Local pixel notation of flag map

Three functions are used in single pass thinning. They are the previous neighbourhood function PN, the current neighbourhood function $\mathrm{CN}$ and the " $0 \rightarrow 1$ " transition function Trans. The previous neighbourhood function PN is defined as follows:

$$
P N\left(P_{0}\right)=\sum_{i=1}^{8} P_{i}
$$

It counts the number of previous neighbours of pixel $\mathrm{P}_{0}$, that is the number of its neighbours in the bitmap from the last iteration. Previous neighbourhood function $\mathrm{PN}$ is used to detect whether a foreground pixel $\mathrm{P}_{0}$, whose pixel value in the bitmap is one, is a boundary point. If $\mathrm{PN}\left(\mathrm{P}_{0}\right)$ is equal to 8 , then pixel $\mathrm{P}_{0}$ is not a boundary point since there is no background pixel, whose pixel value is zero, in its neighbourhood. The current neighbourhood function, $\mathrm{CN}$ is defined as follows,

$$
C N\left(P_{0}\right)=\sum_{i=1}^{8}\left(P_{i} \times Q_{i}\right)
$$

It counts the number of current neighbours of pixel $\mathrm{P}_{0}$. If one foreground pixel in the neighbourhood of $\mathrm{P}_{0}$ has been flagged then it can no longer be considered to exist. The operator " $x$ " in the function $\mathrm{CN}$ is the logical "and' and the operator "+" is the logical "or".

The " $0 \rightarrow 1$ " transition function, Trans, is defined as follows,

$$
\operatorname{Trans}\left(P_{0}\right)=\sum_{i=1}^{8} \operatorname{count}\left(P_{i}\right)
$$

where

$\operatorname{count}\left(P_{i}\right)=\left\{\begin{array}{l}1, \text { if }\left(\left(P_{i} \times Q_{i}=0\right) \& \&\left(P_{i+1} \times Q_{i+1}\right)=1\right) \\ 0, \text { otherwise }\end{array}\right.$

and

$P_{9}=P_{1}, Q_{9}=Q_{1}$

It gives the number of " $0 \rightarrow 1$ " transitions when traversing across the 8-neighbours $\mathrm{P}_{1}, \mathrm{P}_{2}, \ldots, \mathrm{P}_{8}$. The function Trans is used to measure the connectivity within the immediate neighbourhood of the pixel. If the condition $\left(\operatorname{Trans}\left(\mathrm{P}_{0}\right)=1\right)$ and $\left(\mathrm{CN}\left(\mathrm{P}_{0}\right)>1\right)$ are true, then there exists only one connected component within the perimeter of the $3 \times 3$ sub-image. It is safe to remove the central pixel that has the value 1 , since its removal will not affect the connectivity of the rest of the pixels in the local window. If the condition $\left(\operatorname{Trans}\left(\mathrm{P}_{0}\right)=\operatorname{minCN}\left(\mathrm{P}_{0}\right), 8-\left(\mathrm{CN}\left(\mathrm{P}_{0}\right)\right)\right)$ is true, that means 
the central pixel $\mathrm{P}$ is a break point. The removal of the break point will damage the connectivity of the image and hence it must be preserved. Figure 10 shows the thinned image.

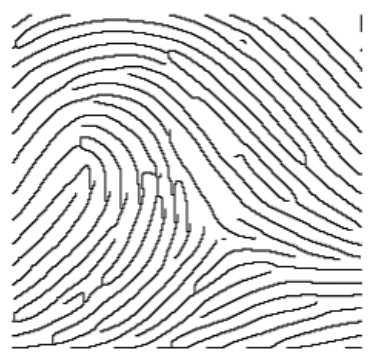

Figure 10.Thinned Image

\section{FINGERPRINT MATCHING}

For the identification process, the input image is preprocessed and thinned to one pixel thick image. From the thinned image, the features points are extracted and matched with the image in database.

Distance matching has been used in the proposed system for the purpose of recognition. Relative distances of each feature point are compared with the stored feature points. The feature point as well as the skeleton image which best matches with the query image and distance value is below the maximum threshold, is recognized as a genuine attempt, else an imposter.

To ensure the validity of the extracted fingerprint, the histogram of fingerprint are developed and compared. Histogram matching of is performed on fingerprint image extracted from database and the input image.
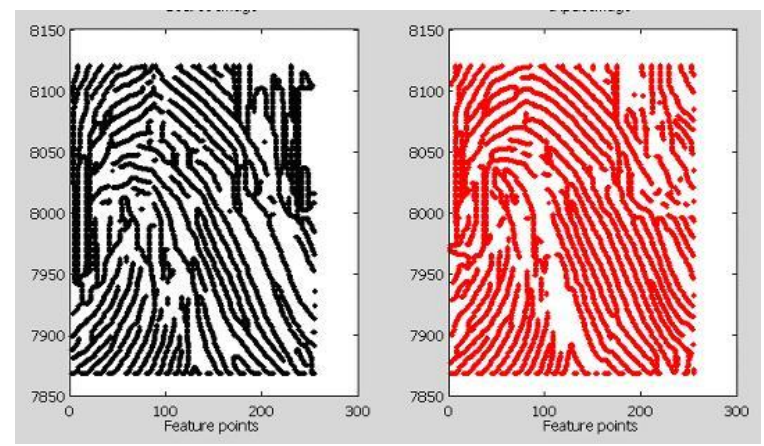

(a)

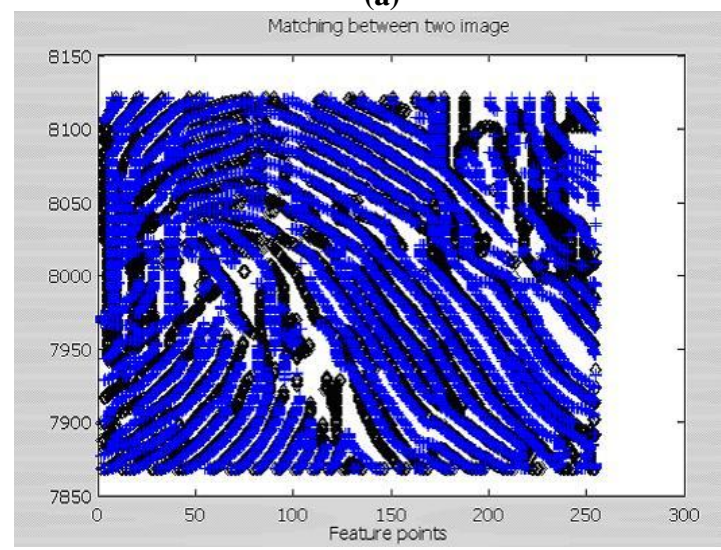

(b)

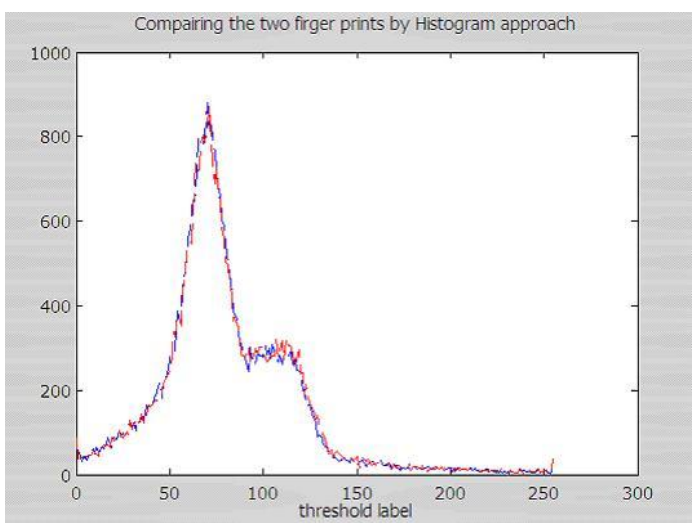

(c)

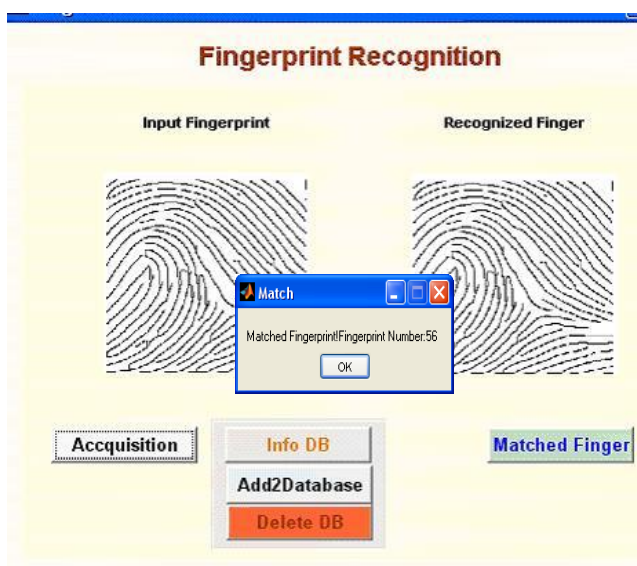

(d)

Figure 11. (a)Feature points images extracted from thinned image (b) Distance Matching between input and source images (c) Comparison using histogram matching (d) Display the extracted skeleton fingerprint

\section{EXPERIMENTAL RESULTS}

In this research work, the proposed matching technique was tested on the database of 300 images from NRC card and FVC2000 database. Each fingerprint image is manually cropped around the center point and enhanced. The skeleton image is extracted and stored into the database. The experimental results show that the cropping area is very important. Cropping around the center area that can have important ridge line information is done for registration and identification. If the cropped areas of the registered image and input image are different, this can lead to a few error rates. But the singularities such as core points to use as a reference point cannot be detected because the fingerprints from NRC cards are very noisy with fabric background. Therefore, the center area around the core point is cropped with the size of $200 \times 200$.

To verify the system, the receiver operating characteristic (ROC) curve and equal error rate (EER) are used to evaluate the performance of the proposed method. The ROC curve is a false acceptance rate (FAR) versus false rejection rate (FRR) curve. For a given distance threshold, FRR and FAR can be calculated. 
Different thresholds are taken to perform tests according to the proposed method and multiple sets of FRR and FAR values are obtained; the FRR and FAR graph of relation is drawn. Figure 12 represents the ROC results, which measures the accuracy of fingerprint matching process and shows the overall performance of an algorithm. The EER is the point where a false acceptance rate and the false rejection rate are equal in value. The smaller the EER is, the better the algorithm. From Figure 12, we can see that the performance of our algorithm is good and the EER is $2.80 \%$ for our own database and EER with $0.05 \%$ for FVC 2000 database. The overall performance of the proposed system is up to $98 \%$.

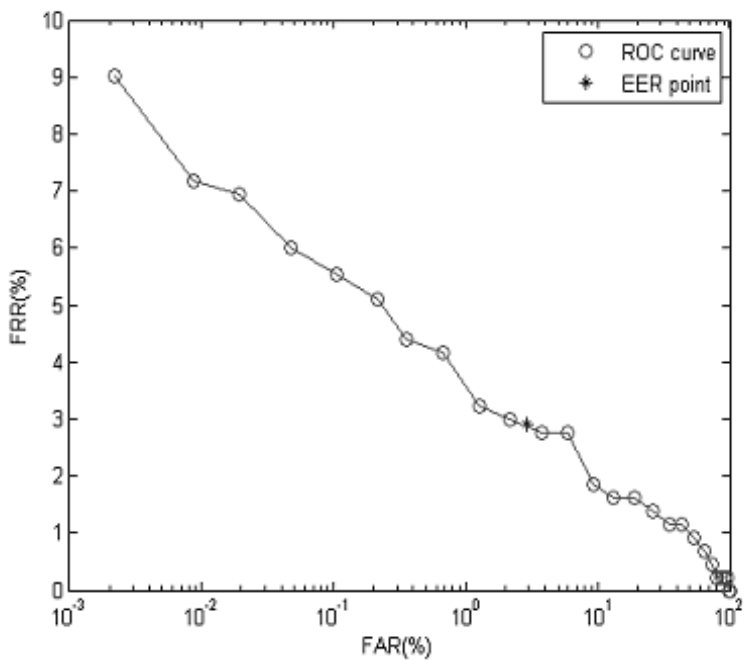

(a)

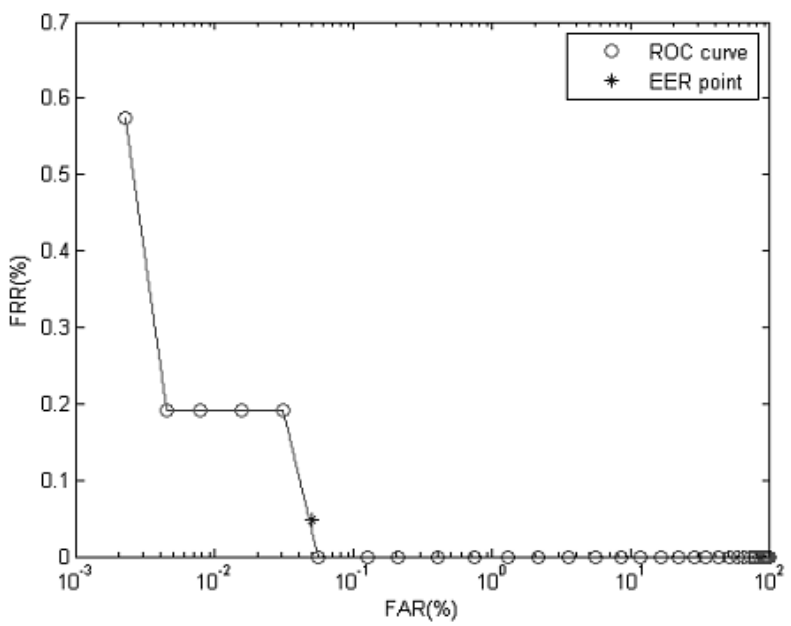

(b)

Figure 12. ROC curves (a) ROC curve on Our DB, (b) ROC curve on FVC2000

\section{CONCLUSION}

This paper proposes a novel method to identify enhanced fingerprint images using single pass thinning to compute the fingerprint features. The results show that the partial fingerprint that has important ridge information can be identified by using the skeleton image. Again, the extracted fingerprint using skeleton matching is matched using histogram matching approach. Therefore, the accuracy of the proposed matching system is effective and efficient for identifying the low quality fingerprint images on both live-scan and inked impression on paper.

\section{REFERENCES}

[1] Prabhakar, S., Pankanti, S. and Jain A.K. 2003.Biometrics recognition: security and privacy concern. IEEE Security\&Privacy Magazine 1, 33-42.

[2] Jain, A. K., Yi C. and Demirkus, M. Jan 2007. Pores and ridges: High resolution fingerprint matching using Level 3 features. IEEE Transactions on Pattern Analysis and Machine Intelligence. vol. 29, no.1, pp. 1-13.

[3] Hong, L. and Jain, A.K.1999. Classification of fingerprint images. 11th Scandinavian Conference on Image Analysis.

[4] Kawagoe, M. and Tojo,A. 1984. Fingerprint pattern classification. Pattern Recognition, pp.295-303.

[5] Jain, A.K., Prabhakar, S., Hong, L. and Pankanti, S.2000. Filterbank-based fingerprint matching. IEEE Trans. Image Process, pp.846-859.

[6] Jain A.K. and Pankanti, S.2000. Fingerprint classification and matching, in: Handbook for Image and Video Processing. Academic Press.

[7] Nilsson, K. and Bigun, J. 2002. Complex filters applied to fingerprint images detecting prominent symmetry points used for alignment. Biometric Authentication, in: LNCS, vol. 2359, Springer-Verlag, Berlin, Heidelberg, pp. 39-47.

[8] Yang, J.C. and Park, D.S. 2008, A fingerprint verification algorithm using tessellated invariant moment features. Neurocomputing, pp.1939-1946.

[9] Jain A.K. and Feng, J. 2010. Latent Fingerprint Matching. IEEE Transs. on Pattern Analysis and Machine Intelligence.

[10] Ito, K., Morita, A., Aoki, T., Higuchi, T., Nakajima, H. and Kobayashi, K.2005. A Fingerprint Recognition Algorithm Using Phase-Based Image Matching for Low-Quality Fingerprints. IEEE Int'l Conf. on Image Processing, vol.II.

[11] LinLin, S. and Alex, K. 2009.A New Wavelet Domain Feature for Fingerprint Recognition. Biomedical Soft Computing and Human Sciences, Vol.14, No.1, pp.55-59.

[12] Tico, M., Kuosmanen, P. and Saarinen, J. 2001.Wavelet Domain Features for Fingerprint Recognition. Electronic Letters, Vol. 37, No. 1, pp. 21-22.

[13] He, Y., Tian, J., Luo, X. and Zhang, T. 2003. Image Enhancement and Minutia Matching in Fingerprint Verification, Pattern Recognition Letter, Vol.24, No. 9-10, pp. 1349-1360.

[14] Maio, D. and Maltoni, D. 1997. Direct Gray-Scale Minutiae Detection in Fingerprints. IEEE Transaction on PAMI, Vol. 19, No. 1, pp.17-40.

[15] Kamijo M. 1993. Classifying Fingerprint Images using Neural Network: Deriving the Classification State, IEEE International Conference on Neural network, vol.3, pp. 19321937. 
[16] Karu K., and Jain A.K. 1996. Fingerprint Classification. Pattern Recognition, vol. 29, no. 3, pp. 389-404.

[17] Dadgostar, M., Tabrizi, P. R., Fatemizadeh, E. and Soltanian Z. H.2009.Feature Extraction using Gabor Filter and Recursive Fisher Linear Discriminant with Application in Fingerprint Identification. Seventh International Conference on Advances in Pattern Recognition, pp. 217-220.

[18] Umair, M. K., Shoab, A. K., Naveed E. and Riaz U. R. 2009.A Fingerprint Verification System using Minutiae and Wavelet Based Features. International Conference on Emerging Technologies, pp. 291-296.

[19] Zhou, W., Han, J., Zeng, X., and Yan, W. 2009.Fingerprint Verification Based on Wavelet and Edge Detection. NinthInternational Conference on Electronic Measurement and Instruments, pp. 1001- 1004.

[20] Leon, Sanchez, J., Aguilar, G., Toscano, G., Perez, L. and Ramirez, H. 2009.Fingerprint Verification Applying Invariant Moments. Fifty Second IEEE International Midwest Symposium on Circuits and Systems, pp. 751 757.

[21] Bhowmik, U. K., Ashrafi, A., and Adhami, R. R. 2009. A Fingerprint Verification Algorithm using the Smallest Minimum Sum of Closest Euclidean Distance. International Conference on Electrical Communications and Computers, pp. $90-95$.

[22] Helfroush, M.S. and Mohammadpour, M. 2009.Fingerprint Verification System: A Non-Minutiae Based Approach.
International Conference on Computer, Control and Communication, pp. $1-4$.

[23] Paulino, A. A., Jain, A. K. and Jianjiang, F., 2010. Latent Fingerprint Matching: Fusion of Manually Marked and Derived Minutiae. $23^{\text {rd }}$ SIBGRAPI- Conference on Graphics, Patterns and Images

[24] Drets, G.A. and Liljenstrom, H.G.1999. Fingerprint subclassification: A neural network approach. Intelligent Biometric Techniques in Fingerprint and Face Recognition, pages 109-134.

[25] OGorman, L. and Nickerson, J.V. 1989. An approach to fingerprint filter design. Pattern Recognition, 22(1):29-38.

[26] Wilson, C.L., Candela, G.T. and Watson, C.I. 1994. Neuralnetwork fingerprint classification. Journal of Artificial Neural Networks, 1(2):203-228.

[27] Kass, M. and Witkin, A.1987. Analyzing oriented patterns. Computer Vision, Graphics, and Image Processing, 37(3):362-385.

[28] Hong, L., W. Y., and Jain. A. K.1998. Fingerprint image enhancement: Algorithm and performance evaluation. IEEE Trans. On Pattern Analysis and Machine Intelligence.

[29] Bazen, A.M. and Gerez, S.H.2002. Systematic methods for the computation of the directional fields and singular points of fingerprints. IEEE Transaction On PAMI, 24(7):905-919.

[30] Ng, G. S., Zhou, R. W. and Quek, C. 1994. A Novel Single Pass Thinning Algorithm, IEEE Transaction on System Man and Cybernetics. 